\title{
Realizing Spin-Hamiltonians in Nanolaser Lattices
}

\author{
M. Parto ${ }^{1, \dagger, *}$, W. Hayenga ${ }^{1,3, \dagger}$, A. Marandi ${ }^{2}$, D. N. Christodoulides ${ }^{1}$, and M. Khajavikhan ${ }^{1,3}$ \\ 1 CREOL, College of Optics and Photonics, University of Central Florida, Orlando, Florida 32816-2700, USA \\ 2 Department of Electrical Engineering, California Institute of Technology, Pasadena, CA 91125, USA \\ 3 Ming Hsieh Department of Electrical and Computer Engineering, University of Southern California, Los Angeles, California 90089, USA \\ *mparto@knights.ucf.edu
}

\begin{abstract}
We demonstrate both theoretically and experimentally that coupled metallic nanolasers can be used to emulate spin-Hamiltonians. Depending on the geometry of the array, we observe ferromagnetic and antiferromagnetic behaviors, as well as geometric frustration. $\odot 2020$ The Author(s) OCIS codes: (140.0140) Lasers and laser optics; (190.4390) Nonlinear optics, integrated optics
\end{abstract}

Spin-Hmailtonians arise in a ubiquitous manner in nature. Over the years, such models have been a subject of intense investigation in many and diverse areas of science and technology, ranging from condensed matter physics [1] and spintronics [2] to quantum information theory [3]. In recent years, there has been a number of attempts to cast various computational optimization problems in terms of finding the ground state of a corresponding spin-Hamiltonian [4]. In this regard, active optical configurations provide an attractive approach for physically implementing and effectively studying such spin systems. In contrast to other schemes that rely on classical or quantum annealing, optical schemes can quickly converge to the global minimum loss, once gain is introduced. This interesting property has incited a flurry of activities in designing photonic "machines" capable of emulating spin exchange Hamiltonians such as the classical Ising or XY Hamiltonians. So far, such active spin systems have been implemented using optical parametric oscillators [5], polaritonic arrangements [6], and degenerate laser cavities [7]. Of interest will be to open up new possibilities by introducing additional degrees of freedom through the vectorial nature of the electromagnetic modes in ultra-small spin-like optical resonant structures.

In this work, we show that the interactions among resonant vectorial modes in coupled metallic nanolaser arrays $[8,9]$ can be used to emulate spin-Hamiltonians such as for example the classical XY Hamiltonian. By properly designing the array elements, two regimes of exchange coupling can be identified, namely a ferromagnetic (FM) and an antiferromagnetic (AF) phase (Fig. 1 (a)). Information obtained from the diffraction patterns as well as the winding numbers associated with the optical vortices emitted by the structures is used to experimentally characterize these phases. Depending on the type of the vectorial resonant modes involved, in the AF state, we demonstrate geometrical frustration in various scenarios, in full accord with theoretical predictions.

(a)

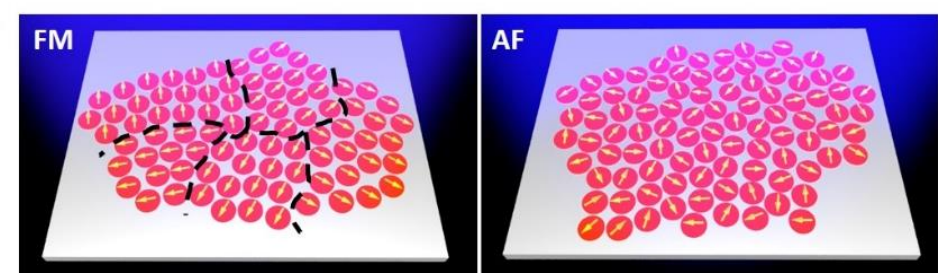

(b)

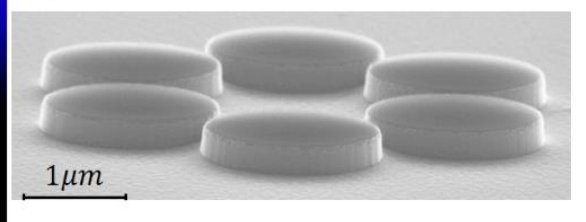

Figure 1: (a) Spin-like behavior in coupled metallic nanolaser arrays with ferromagnetic (FM) and anti-ferromagnetic (AF) exchange interactions. (b) SEM image of an array of six coupled active nanodisks (before silver deposition) used in this study.

To illustrate the spin-like behavior in metallic nanolaser arrays, we consider a circular array of $N$ identical metallic nanodisk lasers, as depicted in the SEM image of Fig. 1 (b) for $N=6$. Here, the coupling between the nearest-neighbor cavity elements takes place via their overlapping near-fields. In the weak coupling regime, one can obtain the transverse distribution of the resonant electromagnetic fields within the nanodisk $j$ from the corresponding longitudinal component of the electric (magnetic) field of the associated TM (TE) mode, i.e. $\psi_{j} \propto$ $J_{n}\left(k_{\rho} \rho\right) \cos \left(n \phi+\phi_{j}\right)$. Here the integer $n$ denotes the azimuthal mode number, while $\phi_{j}$ is the relative phase with respect to the local coordinates at each site. In such an arrangement, the metallic cladding leads to a mode-dependent dissipation $\mathcal{P}_{L}$. The resulting lasing supermodes supported by this lattice can then be found by minimizing this total loss function, which defines the energy landscape of the system. Equivalently, the extrema of such an energy landscape function correspond to the minimum energy eigenstates of an associated Hamiltonian $\mathcal{H}=\mathcal{H}_{\mathrm{XY}}+\mathcal{H}_{0}$ :

$$
\begin{gathered}
\mathcal{H}_{\mathrm{XY}}=\sum_{j=1}^{N} J_{j, j+1} \vec{\sigma}_{j} . \vec{\sigma}_{j+1}, \\
\mathcal{H}_{0}=\sum_{j=1}^{N} J_{0 j, j+1} \cos \left[\phi_{j}+\phi_{j+1}+2 j \times 2 n \pi / N\right] .
\end{gathered}
$$


Here $\mathcal{H}_{\mathrm{XY}}$ is the isotropic $\mathrm{XY}$ Hamiltonian describing exchange interactions between the ensuing classical pseudospins defined in each laser cavity as $\vec{\sigma}_{j}=\left(\cos \phi_{j}, \sin \phi_{j}\right)$, while $\mathcal{H}_{0}$ represents an anisotropic Hamiltonian component responsible for lifting the continuous $U(1)$ symmetry within individual cylindrical disks. The Hamiltonian presented in Eq. (1) can give rise to a variety of field patterns corresponding to ferromagnetic-like (FM) or antiferromagneticlike (AF) interactions between neighboring pseudospins $\vec{\sigma}_{j}$. For instance, in a simple two-element arrangement, a resonant $T E_{22}$ mode within nanodisk elements leads to a lasing supermode with oppositely aligned pseudospins, corresponding to an FM-like coupling between the neighboring array elements $J_{j, j+1}<0$. On the other hand, for a similar configuration albeit with a slightly different size, once the $T E_{14}$ lasing mode dominates, the underlying coupling becomes positive $\left(J_{j, j+1}>0\right)$, as expected from an AF Hamiltonian. When dealing with larger lattices, the AF coupling condition can lead to more complex ground states. In this respect, the competing interactions arising from various nearest-neighbor couplings can result in a scenario where the anti-aligned solution is prohibited from extending across the entire structure due to geometrical constraints. Consequently, the system will eventually minimize its overall energy (loss) by settling into a geometrically frustrated state. Figure 2 (a) shows such a scenario in an AF kagome lattice, where a possible ground state is depicted. Figure 2 (b) presents the lasing supermode in a kagome nanolaser array, as obtained from FEM simulations. To probe long-range order effects in our nanolaser platform, we fabricated two sets of large kagome lattices involving AF-coupled nanolasers with $20 \times 20$ and $40 \times 40$ unit cells (a total of $\sim 1200$ and $\sim 5000$ coupled nanodisk lasers, respectively). As shown in Fig. 2 (c), for the smaller lattice, the far-field diffraction profile exhibits a sharp hexagonal pattern - signifying a fully ordered lasing supermode in this particular system. However, for the larger structure ( $40 \times 40$ unit cells), we observed a noticeable blurring in the associated far-field diffraction peaks (Fig. 2 (d)). The latter result clearly demonstrates an absence of long-range order in the associated pseudospins, akin to that encountered in Heisenberg kagome antiferromagnets.

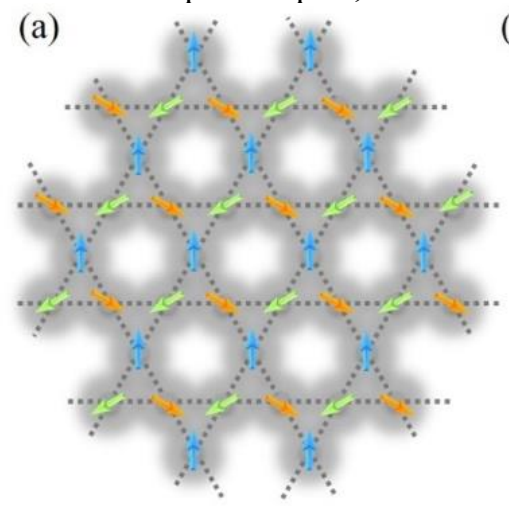

(b)



(c)

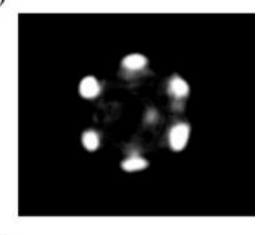

(d)



Figure 2: (a) The pseudospin arrangement of a possible ground state in a kagome lattice. (b) Simulation results of the lasing supermode corresponding to (a). Measured far-field diffraction patterns from a (c) $20 \times 20$ and (d) $40 \times 40$ unit-cell nanolaser kagome lattice.

In conclusion, we demonstrated the first implementation of spin-Hamiltonians in optical nanolaser lattices. We observed various regimes of ferromagnetic and antiferromagnetic coupling, as well as geometric frustration, by appropriately tailoring the geometry of the arrays. Our results could pave the way towards emulating more complex networks and material systems wherein a wide class of optimization problems and phase transition phenomena can be studied.

Acknowledgments. Army Research Office (ARO) (W911NF-16-1-0013, W911NF-17-1-0481), DARPA (D18AP00058, HR00111820042, HR00111820038), National Science Foundation (ECCS 1454531, DMR-1420620, ECCS 1757025, CBET 1805200), Office of Naval Research (N0001416-1- 2640, N00014-18-1-2347), Air Force Office of Scientific Research (FA9550-14-1- 0037), and U.S.-Israel Binational Science Foundation (BSF) (2016381).

\section{References}

[1] J. H. Van Vleck, The Theory Of Electric And Magnetic Susceptibilities, Oxford At The Clarendon Press (1932).

[2] S. A. Wolf et al, "Spintronics: A Spin-Based Electronics Vision for the Future," Science 294, 1488 (2001).

[3] M. W. Johnson et al, "Quantum annealing with manufactured spins," Nature 473, 194 (2011).

[4] G. D. Cuevas, and T. S. Cubitt, "Simple universal models capture all classical spin physics," Science 351, 1180 (2016).

[5] A. Marandi et al, "Network of time-multiplexed optical parametric oscillators as a coherent Ising machine," Nat. Photonics 8, 937 (2014).

[6] N. G. Berloff et al, "Realizing the classical XY Hamiltonian in polariton simulators," Nat. Materials 16, 1120 (2017).

[7] M. Nixon et al, “Observing Geometric Frustration with Thousands of Coupled Lasers," Phys. Rev. Lett. 110, 184102 (2013).

[8] M. Khajavikhan et al, "Thresholdless nanoscale coaxial lasers," Nature 482, 204 (2012).

[9] W. Hayenga et al, "Second-order coherence properties of metallic nanolasers," Optica 3, 1187 (2016). 